Segre. Consider the two quadrics of Moutard belonging to the tanents $t$ and $t^{\prime}$ at a point of the surface; they intersect in the asymptotic tangents and a conic. The limiting position of the plane of this conic as $t^{\prime} \rightarrow t$ is

$$
\begin{aligned}
z\left[4 \beta^{2}-4 \gamma^{2} n^{6}-2 \beta n \phi+\right. & \left.2 \gamma n^{5} \psi+4 \beta \psi n^{2}-4 \gamma \phi n^{4}\right] \\
+ & 8\left(\gamma n^{8}+\beta\right) n^{2} x-8\left(\beta+\gamma n^{3}\right) n y=0,
\end{aligned}
$$

which envelopes the cone of Segre.

National University of Chekiang

\title{
ON A REPRESENTATION IN SPACE OF GROUPS OF CIRCLE AND TURBINE TRANSFORMATIONS IN THE PLANE
}

\section{J. M. FELD}

1. Introduction. In a previous paper [6] the author showed that the oriented lineal elements in the euclidean plane can be mapped continuously and $(1,1)$ upon the points of quasi-elliptic ${ }^{2}$ three-space $Q_{3}$ so that the whirl-similitude group of turbine ${ }^{3}$ transformations in the euclidean plane is represented isomorphically upon the group of projective automorphisms of $Q_{3}$. By means of this representation proper turbines in the plane are mapped upon those real lines in $Q_{3}$ which do not intersect a line $L$, the real part of the quasi-elliptic absolute. It is the purpose of this note to investigate this representation analytically and to extend it so as to yield a $(1,1)$ continuous mapping of the turbines (proper and improper) in the Moebius plane upon all the lines in projective $S_{3}$. By such means we establish the isomorphism between certain groups of projective transformations in space on the one hand, and on the other of Kasner's 15-parameter group of turbine transformations [7] and some of its important subgroups, namely the Moebius, Laguerre, and Lie groups of circle transformations.

Other representations of turbines in space are due to Kasner and DeCicco [7, 8] and to A. Narasinga Rao [9]. The former use a

Presented to the Society, February 26, 1944; received by the editors April 17, 1944.

1 The numbers in brackets refer to the bibliography at the end of this paper.

2 The term quasi-elliptic space is due to Blaschke $[1,2]$. The absolute of this space is composed of a pair of conjugate imaginary planes $x_{3}^{2}+x_{4}^{2}=0$ and a pair of conjugate imaginary points $(1: \pm i: 0: 0)$.

${ }^{3}$ The geometry of turbines was initiated by Kasner [7]. An extensive bibliography on the subject is to be found in [5]. 
representation of lineal elements upon the points of $S_{\mathbf{3}}$, similar to that used here; the latter maps turbines in the plane upon the points of $S_{4}$, and shows thereby that Kasner's group and its subgroups are isomorphic with certain groups of projectivities in $S_{4}$. Kasner and DeCicco do not indicate how their representation would map the groups of Moebius, Laguerre, and Lie in $S_{\mathbf{3}}$.

2. The mapping $\mathfrak{M}$. We shall need the following definitions.

Definitions. A proper turbine is a series of $\infty^{1}$ oriented lineal elements the points of which lie on a cycle (oriented circle) and the directions of which are inclined at the same angle to the direction of the cycle.

An improper turbine is a series of $\infty^{1}$ oriented parallel lineal elements the points of which lie on a straight line.

A proper flat field is a set of $\infty^{2}$ lineal elements which lie on the $\infty^{1}$ cycles having a given lineal element (the center of the flat field) in common.

An improper flat field is a set containing all of the $\infty^{2}$ lineal elements parallel to a given direction.

The proper turbines include the point turbines (= point cycles); the improper turbines include the spears (=oriented lines).

Let the oriented lineal element $\mathrm{e}$ passing through the point $x, y$ in the $x y$-plane have the direction $\theta$. Let $z=x+i y, \bar{z}=x-i y, \zeta=e^{i \theta}$, $0 \leqq \theta<2 \pi$. Let the symbol $(z, \zeta)$ represents $\mathrm{e} ; z$ and $\zeta$ shall be called the coordinates of $e$. In terms of these coordinates a proper turbine has an equation [6] of the form

$$
z-l=r \zeta \text {. }
$$

The point $l$ is the center of the turbine and $|r|$ is the length of its radius. We shall represent the turbine $(2.1)$ by the symbol $[l, r]$; $l, r$ shall be called its coordinates. Turbines $[l, r]$ such that $r+\bar{r}=0$ are cycles, and those such that $r=0$ are point turbines.

A proper flat field having the lineal element $(s, \sigma)$ for its center is given by the equation [6]

$$
z-s=(\bar{z}-\bar{s}) \sigma \zeta .
$$

Let the symbol $\{s, \sigma\}$ represent this flat field. Two proper flat fields $\{s, \sigma\}$ and $\left\{s^{\prime}, \sigma^{\prime}\right\}$ such that $\sigma \neq \sigma^{\prime}$ determine a proper turbine $[l, r]$ where

$$
l=\left(s \sigma^{\prime}-s^{\prime} \sigma\right) /\left(\sigma^{\prime}-\sigma\right), \quad r=\left(z-z^{\prime}\right) /\left(\bar{\sigma}^{\prime}-\bar{\sigma}\right) .
$$

Let a point $\mathfrak{p}$ in $S_{3}$ have the homogeneous coordinates $x_{1}: x_{2}: x_{3}: x_{4}$. 
The representation $\mathfrak{M}$ mapping $(z, \zeta) \rightleftarrows \mathfrak{p}$ continuously and $(1,1)$ is given by the equations

$$
z=2\left(x_{1}+i x_{2}\right) /\left(x_{4}+i x_{8}\right), \quad \zeta=\left(x_{4}-i x_{8}\right) /\left(x_{4}+i x_{3}\right)
$$

and

$$
\begin{array}{ll}
\rho x_{1}=i(z \bar{\xi}+\bar{z}), & \rho x_{2}=z \bar{\xi}-\bar{z}, \\
\rho x_{3}=2(\xi-1), & \rho x_{4}=2 i(1+\xi) .
\end{array}
$$

These equations define $\mathfrak{M}$ for all lineal elements in the euclidean plane and all points in $S_{3}$ except those on the line $L: x_{3}=x_{4}=0$. The points of any plane $u_{i} x_{i}=0$ such that $u_{2}+i u_{1} \neq 0$, that is, a plane that does not pass through $L$, are mapped upon the lineal elements of a proper flat field $\{s, \sigma\}$ where

$$
\begin{aligned}
s=-2\left(u_{3}+i u_{4}\right) /\left(u_{2}+i u_{1}\right), & \sigma=\left(u_{2}-i u_{1}\right) /\left(u_{2}+i u_{1}\right), \\
\rho u_{1}=2 i(1-\bar{\sigma}), & \rho u_{2}=2(\bar{\sigma}+1), \\
\rho u_{3}=-(s \bar{\sigma}+\bar{s}), & \rho u_{4}=i(s \bar{\sigma}-\bar{s}) .
\end{aligned}
$$

Any plane $u$ that does pass through $L$ is mapped upon an improper flat field composed of $\infty^{2}$ parallel lineal elements $(z, \zeta)$ where

$$
\zeta=\left(u_{3}+i u_{4}\right) /\left(u_{3}-i u_{4}\right) .
$$

Let $g$ be any line in $S_{3}$ except $L$. Let the Plücker axial coordinates of $g$ be $q_{i j}=u_{i} v_{j}-u_{j} v_{i}$. Let us assume, as we may, that neither of the planes $u$ and $v$ passes through $L$. Evidently $g$ is mapped by $\mathfrak{M}$ upon a series of $\infty^{1}$ lineal elements common to the proper flat fields $\{s, \sigma\}$ and $\left\{s^{\prime}, \sigma^{\prime}\right\}$ which correspond respectively to $u$ and $v$. Such a series is a proper turbine when $\sigma \neq \sigma^{\prime}$ and an improper turbine when $\sigma=\sigma^{\prime}{ }^{4}$ Consequently, the proper turbines in the euclidean plane correspond $(1,1)$ to those real lines in $S_{3}$ which do not intersect $L$, and the improper turbines correspond $(1,1)$ to the real lines in $S_{3}$ that do intersect $L$; line $L$ itself so far has no $\mathfrak{M}$-image. The correspondence between turbines and lines is given by the equations:

$$
\begin{aligned}
& \rho g_{12}=4 i\left(\bar{\sigma}^{\prime}-\bar{\sigma}\right), \\
& \rho g_{13}=-i\left[\left(s-s^{\prime}\right) \bar{\sigma} \bar{\sigma}^{\prime}-\left(\bar{s}-\bar{s}^{\prime}\right)+\left(\bar{s}+s^{\prime}\right) \bar{\sigma}^{\prime}-\left(s+\bar{s}^{\prime}\right) \bar{\sigma}\right], \\
& \rho g_{14}=-\left(s-s^{\prime}\right) \bar{\sigma} \bar{\sigma}^{\prime}-\left(\bar{s}-\bar{s}^{\prime}\right)+\left(s-\bar{s}^{\prime}\right) \bar{\sigma}-\left(s^{\prime}-\bar{s}\right) \bar{\sigma}^{\prime}, \\
& \rho q_{34}=-i\left(\bar{s} s^{\prime} \bar{\sigma}^{\prime}-s \bar{s}^{\prime} \bar{\sigma}\right), \\
& \rho g_{42}=i\left[\left(s-s^{\prime}\right) \bar{\sigma} \bar{\sigma}^{\prime}-\left(\bar{s}-\bar{s}^{\prime}\right)+\left(s+\bar{s}^{\prime}\right) \bar{\sigma}-\left(\bar{s}+s^{\prime}\right) \bar{\sigma}^{\prime}\right], \\
& \rho g_{28}=\left(s-s^{\prime}\right) \bar{\sigma} \bar{\sigma}^{\prime}+\left(\bar{s}-\bar{s}^{\prime}\right)+\left(s-\bar{s}^{\prime}\right) \bar{\sigma}+\left(\bar{s}-s^{\prime}\right) \bar{\sigma}^{\prime} .
\end{aligned}
$$

${ }^{4} \sigma=\sigma^{\prime}$ if and only if $g$ intersects $L$. 
With the aid of equations (2.3) we find that the proper turbine determined by the flat fields $\{s, \sigma\}$ and $\left\{s^{\prime}, \sigma^{\prime}\right\}$ such that $\sigma \neq \sigma^{\prime}$ is a cycle when $\left(\bar{s}-\xi^{\prime}\right) \sigma \sigma^{\prime}=s-s^{\prime}$, and a point turbine when $s=s^{\prime}$. If $\sigma=\sigma^{\prime}$ the pair of flat fields determines an improper turbine, which, in the event that $\left(\vec{s}-\bar{s}^{\prime}\right) \sigma^{2}=s-s^{\prime}$, becomes a spear. From (2.7) we find that to the cycles and spears in the euclidean plane correspond $(1,1)$ all but one of the lines of the linear complex

A:

$$
q_{13}-q_{42}=0 ;
$$

the excepted line is $L\left(q_{12}: q_{13}: q_{14}: q_{34}: q_{42}: q_{23}=0: 0: 0: 1: 0: 0\right)$. To the point turbines in the euclidean plane correspond $(1,1)$ all the lines but one, namely $L$, of the rotationally symmetric elliptic congruence

B:

$$
q_{13}-q_{42}=0, \quad q_{14}-g_{23}=0,
$$

which has the lines $x_{1}+i x_{2}=x_{3}-i x_{4}=0$ and $x_{1}-i x_{2}=x_{3}+i x_{4}=0$ as directrices.

To make $\mathfrak{M}(1,1)$ for all the lines of $S_{3}$, we therefore close the euclidean plane by adjoining one ideal point turbine at infinity $t_{\infty}$, which shall serve as the $\mathfrak{M}$-image of $L$. To the point turbines of the closed (Moebius) plane there now correspond continuously and $(1,1)$ the lines of $B$, and to the cycles and spears of the Moebius plane there correspond $(1,1)$ the lines of $\mathcal{A}$.

To the improper turbines correspond $(1,1)$ the lines of the special complex $q_{12}=0$; this complex has the line $L$ for its directrix. To the spears, therefore, correspond $(1,1)$ the lines of the special congruence c:

$$
g_{13}-g_{42}=0, \quad g_{12}=0 .
$$

Let the terms Moebius cycle, Laguerre cycle, and Lie cycle designate respectively (1) a cycle with nonzero radius or a spear, (2) any cycle except $t_{\infty},(3)$ any cycle or spear in the Moebius plane. Since the Moebius group of circle transformations in the plane, regarded as an extended group of contact transformations, transforms point turbines into point turbines and Moebius cycles into Moebius cycles, its $\mathfrak{M}$ image in $S_{3}$ is a group of $\infty^{6}$ collineations that transform the congruence $B$ into itself and also transform the linear complex $\mathcal{A}$ into itself. The large (seven-parameter) Laguerre group in the plane is a group of contact transformations that convert spears into spears and Laguerre cycles in to Laguerre cycles; consequently, this Laguerre group is isomorphic with a group of $\infty^{7}$ collineations in $S_{3}$ that leave invariant both the special congruence $\mathcal{C}$ and the complex $\mathcal{A}$. Lie's 10-parameter group of contact transformations transforming Lie cycles into Lie cycles is isomorphic with the group of $\infty^{10}$ collineations in $S_{3}$ 
that leave $\mathcal{A}$ invariant. Kasner's 15 -parameter group of turbine transformations in the plane is isomorphic with the group of projectivities (collineations and correlations) in $S_{8}$.

Various other representations of the Moebius, Laguerre, and Lie groups have been given, for instance in $[3,4]$.

\section{BIBLIOGRAPHY}

1. W. Blaschke, Euklidische Kinematik und nichteuklidische Geometrie, Zeitschrift fur Mathematik und Physik vol. 60 (1911) pp. 61-91 and 203-204.

2. - Ebene Kinematik, Leipzig and Berlin, 1938.

3. - Vorlesungen uber Differentialgeometrie, vol. 3, Berlin, 1929.

4. J. DeCicco, The analogue of the Moebius group of circular transformations in the Kasner plane, Bull. Amer. Math. Soc. vol. 45 (1939) pp. 936-944.

5. - The geometry of fields of lineal elements, Trans. Amer. Math. Soc. vol. 47 (1940) pp. 207-229.

6. J. M. Feld, Whirl-similitudes, euclidean kinematics, and non-euclidean geometry, Bull. Amer. Math. Soc. vol. 48 (1942) pp. 783-790.

7. E. Kasner, Turns, slides and the geometry of turbines, Amer. J. Math. vol. 33 (1911) pp. 193-202.

8. E. Kasner and J. DeCicco, Quadric fields in the geometry of the whirl-motion group $G_{6}$, Amer. J. Math. vol. 61 (1939) pp. 131-142.

9. A. Narasinga Rao, Studies in turbine geometry I, J. Indian Math. Soc. vol. 3 (1938) pp. 96-108; II, Proceedings of the Indian Academy of Sciences vol. 8A (1938) pp. 179-186.

Queens College 